\title{
A multicentre randomised trial to compare the efficacy of omeprazole versus rabeprazole in early symptom relief in patients with reflux esophagitis
}

\author{
Akihito Nagahara $\cdot$ Tsuyoshi Suzuki $\cdot$ Naoyoshi Nagata $\cdot$ Nozomu Sugai $\cdot$ \\ Yoshiaki Takeuchi · Kouichi Sakurai • Masaki Miyamoto $\cdot$ Kazuhiko Inoue • \\ Junichi Akiyama $\cdot$ Katsuhiro Mabe $\cdot$ Ichiro Konuma • Tomoari Kamada • \\ Ken Haruma
}

Received: 15 August 2013/Accepted: 6 December 2013/Published online: 24 December 2013 (C) The Author(s) 2013. This article is published with open access at Springerlink.com

\begin{abstract}
Background Proton pump inhibitors (PPIs) are affected by cytochrome P450 2C19 (CYP2C19) polymorphisms. This study compared the effect of two PPIs on early symptom relief in Japanese patients with reflux esophagitis, classified by the CYP2C19 phenotype.

Methods Patients with reflux esophagitis were randomised to treatment with omeprazole $20 \mathrm{mg}$ or rabeprazole $10 \mathrm{mg}$ once daily. The CYP2C19 phenotype [homozygous extensive metaboliser (homoEM), heterozygous extensive metaboliser (heteroEM) or poor metaboliser (PM)] of each patient was determined. The primary efficacy endpoint was early, sufficient (Global Overall Symptom scale score
\end{abstract}

A. Nagahara $(\bowtie)$

Department of Gastroenterology,

Juntendo University School of Medicine, Tokyo, Japan

e-mail: nagahara@juntendo.ac.jp

T. Suzuki

Department of Gastroenterology,

Tokyo Metropolitan Police Hospital, Tokyo, Japan

N. Nagata $\cdot$ J. Akiyama

Department of Gastroenterology and Hepatology,

National Center for Global Health and Medicine,

Tokyo, Japan

N. Sugai

Department of Gastroenterology, KKR Sapporo Medical Center,

Hokkaido, Japan

Y. Takeuchi

Department of Gastroenterology,

Showa University School of Medicine, Tokyo, Japan

K. Sakurai

Department of Gastroenterology and Hepatology,

Kumamoto University, Kumamoto, Japan
1 or 2), sustained (maintained for $\geq 7$ days) reflux symptom relief.

Results Of the 199 patients included in this analysis, the proportion achieving sufficient, sustained reflux symptom relief was higher with omeprazole than with rabeprazole on day 1 (35.6 vs. $22.4 \% ; p=0.041)$ and day 2 (43.6 vs. $28.6 \% ; p=0.028)$; there was no significant difference between the two groups on days 3-7. Among patients with the CYP2C19 PM phenotype, sufficient, sustained reflux symptom relief was higher with omeprazole than with rabeprazole on days 4-7 (62.5-66.9 vs 31.6 \%; $p \leq 0.03$ ); differences were not significant on days $1-3$, or among those with the homoEM or heteroEM phenotypes on days $1-7$.

M. Miyamoto

Department of General Internal Medicine, Hiroshima Prefectural Hospital, Hiroshima, Japan

\section{K. Inoue}

Department of General Medicine, Kawasaki Medical School,

Okayama, Japan

\section{K. Mabe}

Division of Endoscopy, Hokkaido University Hospital,

Hokkaido, Japan

I. Konuma

Konuma Clinic, Tochigi, Japan

T. Kamada $\cdot$ K. Haruma

Department of Gastroenterology, Kawasaki Medical School, Okayama, Japan 
Conclusions In Japanese patients with reflux esophagitis, omeprazole $20 \mathrm{mg}$ is more effective than rabeprazole $10 \mathrm{mg}$ at achieving early, sufficient, sustained reflux symptom relief in individuals with the CYP2C19 PM phenotype, and is similarly effective to rabeprazole $10 \mathrm{mg}$ in those with heteroEM or homoEM phenotypes.

Keywords Omeprazole $\cdot$ Rabeprazole Proton pump inhibitor · Reflux esophagitis - Symptom relief

\section{Introduction}

Gastroesophageal reflux disease (GERD) is characterised by reflux of the stomach contents and/or bile into the esophagus [1], typically causing symptoms of heartburn and acid regurgitation [2]. Studies conducted in Japan have found that between 6.5 and $9.5 \%$ of the population have reflux symptoms on at least 1 day per week, and the reported prevalence of reflux esophagitis ranges from 4.9 to $8.2 \%$ [3]. Various lifestyle factors are reported to be associated with GERD [4], and reflux symptoms negatively affect health-related quality of life, work productivity, and health resource utilisation [5-8]. Moreover, reflux esophagitis is a risk factor for Barrett's esophagus and esophageal adenocarcinoma [9]. Proton pump inhibitors (PPIs) are the most effective treatment for GERD, including endoscopically confirmed reflux esophagitis $[1,10]$. Most individuals experience resolution of their reflux symptoms when taking a PPI $[1,10]$, with a concomitant overall improvement in health-related quality of life $[11,12]$.

PPIs are metabolised via the hepatic enzyme cytochrome P450 2C19 (CYP2C19). There are three genetic polymorphisms of CYP2C19, resulting in homozygous extensive metaboliser (homoEM), heterozygous extensive metaboliser (heteroEM) and poor metaboliser (PM) phenotypes [13]. These CYP2C19 phenotypes have different effects on the pharmacodynamic and pharmacokinetic profiles of PPIs. Gastric acid secretion is affected such that post-PPI intragastric $\mathrm{pH}$ values are highest in the PM group and lowest in the homoEM group following administration of omeprazole or rabeprazole [14]. The clinical relevance of these differences is especially important for patients in Japan, where the PM phenotype is much more common (prevalence $18.0-22.5 \%$ ) than in the USA or Europe (prevalence $\leq 3.7 \%$ ) [13].

Results from studies in healthy Japanese volunteers suggest that early effects on gastric acid inhibition in people with different CYP2C19 phenotypes may depend on the type of PPI used [15-17]. Compared with omeprazole $20 \mathrm{mg}$ or lansoprazole $30 \mathrm{mg}$, rabeprazole $10 \mathrm{mg}$ has been shown to exert a faster and more pronounced inhibition of gastric acid secretion in healthy Japanese volunteers with the homoEM or heteroEM phenotypes [15]; however, in another study also conducted in healthy Japanese volunteers with the homoEM or heteroEM phenotypes, lansoprazole $30 \mathrm{mg}$ was shown to induce an earlier rise in blood PPI concentration and intragastric $\mathrm{pH}$ than rabeprazole $10 \mathrm{mg}$ [16]. Furthermore, in healthy Japanese volunteers receiving omeprazole $20 \mathrm{mg}$ or rabeprazole $10 \mathrm{mg}$, there was no significant difference between the two PPIs in early intragastric $\mathrm{pH}$ changes in individuals with the homoEM phenotype, but intragastric $\mathrm{pH}$ was significantly higher with omeprazole than with rabeprazole $6-8 \mathrm{~h}$ after PPI administration according to combined data from participants with the heteroEM and PM phenotypes [17]. Thus, whereas the pharmacodynamic and pharmacokinetic profiles of omeprazole and rabeprazole are clearly dependent on CYP2C19 phenotype, data from healthy volunteers on differences in early acid inhibitory effects between the two PPIs are inconsistent. In addition, there is a paucity of data on whether any differences in early acid inhibitory effects in healthy volunteers translate into early differences in clinical outcomes in patients with GERD.

We conducted this study to compare the efficacy of omeprazole $20 \mathrm{mg}$ and rabeprazole $10 \mathrm{mg}$ in achieving early symptom relief in Japanese patients with reflux esophagitis. Our analysis took into consideration outcomes based on CYP2C19 phenotype.

\section{Methods}

Study design

This was a 4-week, multicentre, randomised, open-label, parallel-group study conducted at 18 centres in Japan between January 2010 and March 2011. Eligible patients were randomly allocated by the study coordination centre (five patients per block per study centre) to receive omeprazole $20 \mathrm{mg}$ or rabeprazole $10 \mathrm{mg}$ orally once daily (in the morning) for 4 weeks. Participants were asked to complete the investigator-administered Global Overall Symptom (GOS) scale to determine symptom severity at baseline (screening visit) and at the end of 2 and 4 weeks of PPI therapy. In addition, participants used the GOS scale to record the severity of their reflux symptoms (heartburn and acid regurgitation) in a daily diary (before bedtime) during the first 2 weeks of the study. An overview of the study design is provided in Fig. 1.

Eligible participants were also asked to provide two $5 \mathrm{~mL}$ blood samples at screening to determine their Helicobacter pylori status (assessed using enzyme immunoassay) and CYP2C19 phenotype (homoEM, heteroEM or $\mathrm{PM}$; assessed using gene analysis by fluorescence correlation spectroscopy). 
Omeprazole $20 \mathrm{mg} /$ day $(n=101)$

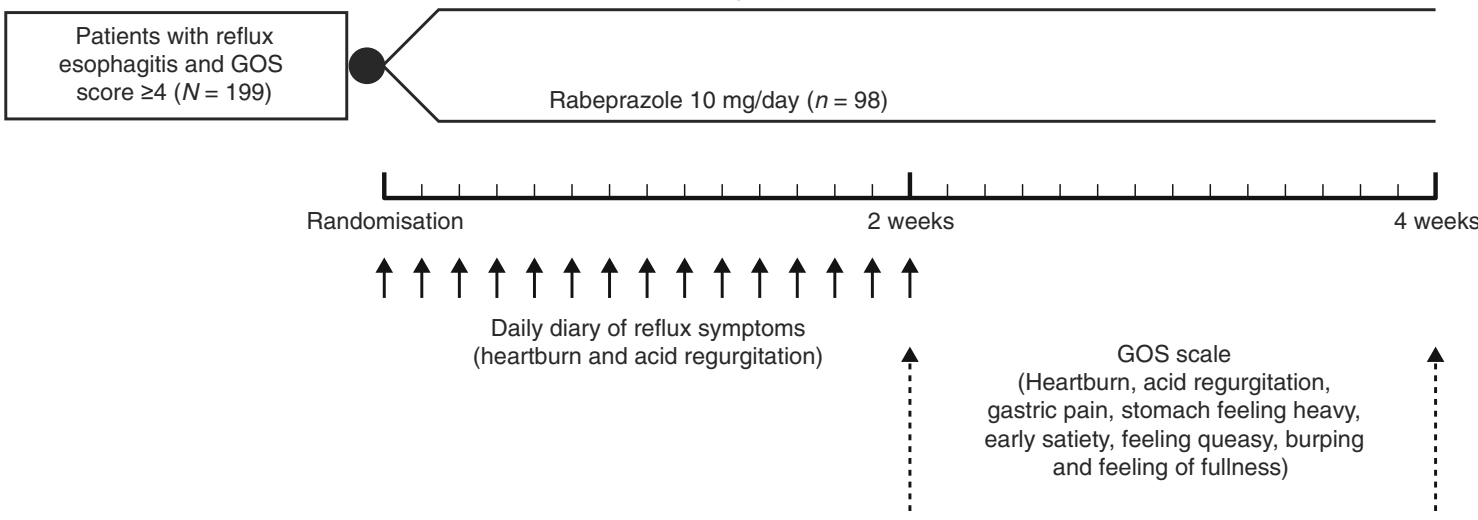

Fig. 1 Overview of study design. GOS Global Overall Symptom

The study protocol was reviewed and approved by the ethics review boards of all participating centres before the start of the investigation. The study was conducted in accordance with the principles of the Declaration of Helsinki and all patients provided informed consent as a condition of participation.

\section{Patients}

Patients of either sex aged 20 years and older were eligible for inclusion if they had diagnoses of reflux esophagitis (Los Angeles grades A-D) on endoscopy during the preceding 12 months. Individuals also had to have heartburn and/or acid regurgitation of at least moderate severity (GOS scale score $\geq 4$ ) at baseline (screening visit).

Exclusion criteria were: 'alarm' features (e.g. vomiting, gastrointestinal haemorrhage and involuntary weight loss); peptic ulcer (other than those at the scarred stage); history of gastrointestinal resection or vagotomy; history of inflammatory bowel disease, irritable bowel syndrome, esophageal stenosis, esophageal achalasia, Zollinger-Ellison syndrome, malabsorption or cerebral disorders; serious hepatic, renal or cardiac disease; confirmed or suspected malignancies; or requirement for continued use of medication that might interact with the test drugs (e.g. atazanavir sulphate, diazepam, phenytoin, warfarin, tacrolimus hydrate, digoxin, methyldigoxin, itraconazole, gefitinib, voriconazole, acid suppressants containing aluminium hydroxide gel, or magnesium hydroxide). Women who were or might have been pregnant, or who were lactating, were also excluded from the study.

The following medications were discontinued at least 1 week before study entry and were not allowed during the study period: PPIs (other than the study PPIs), histamine-2 receptor antagonists, prokinetic agents, gastric mucosal protective agents, anticholinergic drugs, antidepressants, anxiolytics, antidiabetic agents, steroids (other than topical steroids), non-steroidal anti-inflammatory drugs [including acetylsalicylic acid (ASA) preparations and low-dose ASA], and bisphosphonates.

\section{Efficacy assessments}

The efficacy of omeprazole $20 \mathrm{mg}$ and rabeprazole $10 \mathrm{mg}$ was assessed on the basis of the GOS scale heartburn and acid regurgitation scores recorded by patients in their daily diary entries during the first 2 weeks, and from the GOS scale that was completed at the clinic after 2 and 4 weeks of PPI therapy.

The GOS scale has been validated for the assessment of upper gastrointestinal symptoms in the clinical trial setting [18], and has been used in clinical studies to assess symptoms of GERD (heartburn and acid regurgitation) and other upper gastrointestinal symptoms [19-21]. The GOS scale measures the severity of eight symptoms (heartburn, acid regurgitation, gastric pain, stomach feeling heavy, early satiety, feeling queasy, burping and feeling of fullness) on a 7-point scale, from 1 ['no problem' (no symptoms)] to 7 ['very severe problem' (cannot be ignored and markedly limits my daily activities and often requires rest)] [18]. The GOS scale was used in the current study to perform symptom-based evaluations, not to diagnose reflux esophagitis. Therefore, no cut-off value was implemented in this study.

Primary and secondary endpoints

The primary efficacy endpoint was the proportion of patients who had sufficient and sustained (for $\geq 7$ consecutive days) relief of reflux symptoms, defined as the first day of PPI therapy on which the GOS scale score was 1 ['no problem' (no symptoms)] or 2 ['minimal problem (can be easily ignored without effort)']. Secondary efficacy endpoints included the proportion of patients who had: 
sufficient and sustained relief of reflux symptoms assessed by CYP2C19 phenotype; sufficient relief of reflux symptoms (GOS scale score of 1 or 2) after 2 and 4 weeks of PPI therapy (overall and by CYP2C19 phenotype); sufficient relief of upper gastrointestinal symptoms (GOS scale score of 1 or 2) after 2 and 4 weeks of PPI therapy (overall and by CYP2C19 phenotype); complete resolution of reflux symptoms (GOS scale score of 1) after 2 and 4 weeks of PPI therapy (overall and by CYP2C19 phenotype); and complete resolution of upper gastrointestinal symptoms (GOS scale score of 1) after 2 and 4 weeks of PPI therapy (overall and by CYP2C19 phenotype).

\section{Safety assessments}

Adverse events were recorded throughout the study period and were assessed according to whether or not they were serious, their relationship to the study drug, their time of onset, and the outcome. All adverse events were reported descriptively.

\section{Statistical analyses}

\section{Sample size}

The median time to reach heartburn control was estimated to be 2 days for the omeprazole $20 \mathrm{mg}$ group and 3 days for the rabeprazole $10 \mathrm{mg}$ group, based on data by Bytzer et al. [22], and taking into consideration the distribution of the different phenotypes and the treatment doses. Based on these median times, the proportion of patients with symptom improvement was estimated to be $90 \%$ in the omeprazole $20 \mathrm{mg}$ group and $77 \%$ in the rabeprazole $10 \mathrm{mg}$ group. Using these criteria, 97 patients were required to participate in the study to detect a $5 \%$ (two-sided) intergroup difference in the primary variable using the log-rank test. A dropout rate of approximately $10 \%$ was assumed; thus, the plan was to enrol approximately 220 patients (110 per group).

\section{Efficacy and safety variables}

The primary and secondary efficacy endpoints were analysed using data from all patients with at least one assessment of efficacy after the initiation of study treatment.

Sufficient symptom relief was defined as a score of 1 or 2 on the GOS scale, and complete symptom resolution was defined as a score of 1 on the GOS scale. Sufficient and sustained symptom relief was defined as maintenance of sufficient symptom relief for at least 7 consecutive days. Inter-group differences in the proportion of patients reaching the primary and secondary endpoints were analysed using the $\chi^{2}$ test, with statistical significance defined as $p<0.05$ (two-sided).

\section{Results}

Patient population

In total, 209 eligible patients with reflux esophagitis were randomised and received at least one dose of omeprazole $20 \mathrm{mg} \quad(n=106)$ or rabeprazole $10 \mathrm{mg} \quad(n=103)$. Evaluable data were available for 101 patients $(95.3 \%)$ in the omeprazole $20 \mathrm{mg}$ group and 98 patients $(95.1 \%)$ in the rabeprazole $10 \mathrm{mg}$ group. Ten patients $(4.8 \%)$ were excluded from the efficacy analysis because they did not provide daily diary records.

All patients included in this analysis had heartburn and/ or acid regurgitation of at least moderate severity (GOS scale score $\geq 4$ ) at baseline, in accordance with study inclusion criteria. Baseline demographics and clinical characteristics are listed in Table 1; there were no significant differences between the two treatment groups. Mean GOS scale scores at baseline were similar in the two treatment groups (Table 2).

\section{Efficacy}

\section{Primary efficacy endpoint}

On day 1 of PPI therapy, the proportion of patients achieving sufficient and sustained relief of their reflux symptoms for at least 7 consecutive days was $35.6 \%$ with omeprazole $20 \mathrm{mg}$ and $22.4 \%$ with rabeprazole $10 \mathrm{mg}$ ( $p=0.041$; Fig. 2). On day 2 of PPI therapy, it was $43.6 \%$ with omeprazole $20 \mathrm{mg}$ and $28.6 \%$ with rabeprazole $10 \mathrm{mg}(p=0.028$; Fig. 2). Sufficient and sustained relief of reflux symptoms continued to be observed in a greater proportion of patients in the omeprazole $20 \mathrm{mg}$ group (46.5-61.4\%) than in the rabeprazole $10 \mathrm{mg}$ group (39.8-52.0\%) on days 3-7, but the difference between the two groups was no longer statistically significant.

\section{Secondary efficacy endpoints}

Among patients with the PM phenotype, a greater proportion in the omeprazole $20 \mathrm{mg}$ than in the rabeprazole $10 \mathrm{mg}$ group achieved sufficient and sustained relief of their reflux symptoms on days 1-7 of PPI therapy; between-group differences reached statistical significance on days 4-7 (omeprazole 62.5-66.9\% vs rabeprazole $31.6 \% ; p \leq 0.03$; Fig. 3a). Sufficient and sustained relief of reflux symptoms on days 1-7 of PPI therapy was not statistically different in the omeprazole $20 \mathrm{mg}$ and the 
Table 1 Baseline demographics and clinical characteristics

\begin{tabular}{|c|c|c|c|c|}
\hline & Omeprazole $(n=101)$ & Rabeprazole $(n=98)$ & Total $(N=199)$ & $p$ value $^{\mathrm{a}}$ \\
\hline \multicolumn{5}{|l|}{ Sex } \\
\hline Male & $54(53.5)$ & $58(59.2)$ & $112(56.3)$ & \multirow[t]{2}{*}{0.416} \\
\hline Female & $47(46.5)$ & $40(40.8)$ & $87(43.7)$ & \\
\hline \multicolumn{5}{|l|}{ Age, years } \\
\hline 29 & $3(3.0)$ & $2(2.0)$ & $5(2.5)$ & \multirow[t]{7}{*}{0.970} \\
\hline $30-39$ & $11(10.9)$ & $11(11.2)$ & $22(11.1)$ & \\
\hline $40-49$ & $16(15.8)$ & $20(20.4)$ & $36(18.1)$ & \\
\hline $50-59$ & $26(25.7)$ & $23(23.5)$ & $49(24.6)$ & \\
\hline $60-69$ & $25(24.8)$ & $24(24.5)$ & $49(24.6)$ & \\
\hline 70 & $20(19.8)$ & $18(18.4)$ & $38(19.1)$ & \\
\hline Mean \pm SD & $56.8 \pm 11.2$ & $55.8 \pm 11.3$ & $56.3 \pm 13.7$ & \\
\hline \multicolumn{5}{|l|}{ BMI, $\mathrm{kg} / \mathrm{m}^{2}$} \\
\hline$<25$ & $60(59.4)$ & $63(64.3)$ & $123(61.8)$ & \multirow[t]{3}{*}{0.468} \\
\hline$\geq 25$ & $39(38.6)$ & $33(33.8)$ & $72(36.2)$ & \\
\hline Unknown & $2(2.0)$ & $2(2.0)$ & $4(2.0)$ & \\
\hline \multicolumn{5}{|c|}{ Reflux esophagitis, LA grade } \\
\hline A & $55(54.5)$ & $68(69.4)$ & $123(61.8)$ & \multirow[t]{5}{*}{0.220} \\
\hline B & $34(33.7)$ & $23(23.5)$ & $57(28.6)$ & \\
\hline $\mathrm{C}$ & $9(8.9)$ & $6(6.1)$ & $15(7.5)$ & \\
\hline $\mathrm{D}$ & $2(2.0)$ & $1(1.0)$ & $3(1.5)$ & \\
\hline Unknown & $1(1.0)$ & $0(0.0)$ & $1(0.5)$ & \\
\hline \multicolumn{5}{|l|}{ Helicobacter pylori test } \\
\hline Negative & $76(75.2)$ & $65(66.3)$ & $141(70.9)$ & \multirow[t]{4}{*}{0.168} \\
\hline Positive & $9(8.9)$ & $19(19.4)$ & $28(14.1)$ & \\
\hline Judgement impossible & $1(1.0)$ & $2(2.0)$ & $3(1.5)$ & \\
\hline Unknown & $15(14.9)$ & $12(12.2)$ & $27(13.6)$ & \\
\hline \multicolumn{5}{|l|}{ CYP2C19 test } \\
\hline HomoEM & $36(35.6)$ & $37(37.8)$ & $73(36.7)$ & \multirow[t]{4}{*}{0.803} \\
\hline HeteroEM & $40(39.6)$ & $42(42.9)$ & $82(41.2)$ & \\
\hline PM & $23(22.8)$ & $19(19.4)$ & $42(21.1)$ & \\
\hline Unknown & $2(2.0)$ & $0(0.0)$ & $2(1.0)$ & \\
\hline
\end{tabular}

Data presented as $n(\%)$ unless otherwise indicated

$B M I$ body mass index, CYP2C19 cytochrome P450 2C19, heteroEM hetero extensive metaboliser, homoEM homo extensive metaboliser, LA Los Angeles, $P M$ poor metaboliser, $S D$ standard deviation

${ }^{a}$ Excluding unknown and judgement impossible

rabeprazole $10 \mathrm{mg}$ groups in patients with either the homoEM or heteroEM phenotypes (Fig. 3b, c).

After 2 and 4 weeks of PPI therapy, the proportion of patients achieving sufficient relief of their reflux symptoms was similar in the omeprazole $20 \mathrm{mg}$ and rabeprazole $10 \mathrm{mg}$ groups overall (Fig. 4a), and in patients with the homoEM and the heteroEM phenotypes (Fig. 4b). In patients with the PM phenotype, however, a significantly greater proportion in the omeprazole $20 \mathrm{mg}$ group than in the rabeprazole $10 \mathrm{mg}$ group achieved sufficient relief of their reflux symptoms after 2 weeks (78.3 vs $42.1 \%$; $p=0.016)$ and 4 weeks (95.7 vs $68.4 \% ; p=0.018)$ of PPI therapy (Fig. 4b).
Complete resolution of reflux symptoms was achieved in a significantly greater proportion of patients in the omeprazole $20 \mathrm{mg}$ than in the rabeprazole $10 \mathrm{mg}$ group after both 2 weeks (44.0 vs $27.1 \% ; p=0.013)$ and 4 weeks $(55.0$ vs $36.5 \%$; $p=0.009$ ) of PPI therapy (Fig. 5a). When assessed by CYP2C19 phenotype, a significantly greater proportion of patients with the heteroEM phenotype in the omeprazole $20 \mathrm{mg}$ than in the rabeprazole $10 \mathrm{mg}$ group achieved complete reflux symptom resolution after 2 weeks of PPI therapy (43.6 vs $20.0 \% ; p=0.024$ ), but there was no significant difference after 4 weeks of therapy (Fig. 5b). No significant inter-group difference in this measure was noted at either time point for patients with the homoEM or PM phenotype (Fig. 5b). 
Table 2 Baseline Global Overall Symptom scale scores

\begin{tabular}{lllll}
\hline $\begin{array}{l}\text { GOS scale } \\
\text { score }\end{array}$ & $\begin{array}{l}\text { Omeprazole } \\
(n=101)\end{array}$ & $\begin{array}{l}\text { Rabeprazole } \\
(n=98)\end{array}$ & $\begin{array}{l}\text { Total } \\
(N=199)\end{array}$ & $p$ value \\
\hline
\end{tabular}

Heartburn

$\begin{array}{lllll}7 & 4(4.0) & 2(2.0) & 6(3.0) & 0.448 \\ 6 & 13(12.9) & 12(12.2) & 25(12.6) & \\ 5 & 28(27.7) & 24(24.5) & 52(26.1) & \\ 4 & 37(36.6) & 39(39.8) & 76(38.2) & \\ 3 & 8(7.9) & 12(12.2) & 20(10.1) \\ 2 & 8(7.9) & 6(6.1) & 14(7.0) \\ 1 & 3(3.0) & 3(3.1) & 6(3.0) \\ \text { Mean } \pm \mathrm{SD} & 4.3(1.5) & 4.2(0.9) & 4.3(1.3)\end{array}$

Sensation of reflux of gastric acid

\begin{tabular}{|c|c|c|c|c|}
\hline 7 & $6(5.9)$ & $1(1.0)$ & $7(3.5)$ & 0.337 \\
\hline 6 & $11(10.9)$ & $11(11.2)$ & $22(11.1)$ & \\
\hline 5 & $18(17.8)$ & $14(14.3)$ & $32(16.1)$ & \\
\hline 4 & $29(28.7)$ & $35(35.7)$ & $64(32.2)$ & \\
\hline 3 & $16(15.8)$ & $14(14.3)$ & $30(15.1)$ & \\
\hline 2 & $18(17.8)$ & $14(14.3)$ & $32(16.1)$ & \\
\hline 1 & $3(3.0)$ & $8(8.2)$ & $11(5.5)$ & \\
\hline Mean $\pm \mathrm{SD}$ & $4.0 \pm 1.2$ & $3.7 \pm 1.2$ & $3.9(1.5)$ & \\
\hline \multicolumn{5}{|l|}{ Gastric pain } \\
\hline 7 & $4(4.0)$ & $1(1.0)$ & $5(2.5)$ & 0.835 \\
\hline 6 & $5(5.0)$ & $4(4.1)$ & $9(4.5)$ & \\
\hline 5 & $10(9.9)$ & $10(10.2)$ & $20(10.1)$ & \\
\hline 4 & $13(12.9)$ & $15(15.3)$ & $28(14.1)$ & \\
\hline 3 & $16(15.8)$ & $22(22.4)$ & $38(19.1)$ & \\
\hline 2 & $18(17.8)$ & $14(14.3)$ & $32(16.1)$ & \\
\hline 1 & $35(34.7)$ & $31(31.6)$ & $66(33.2)$ & \\
\hline Mean $\pm \mathrm{SD}$ & $2.8 \pm 1.5$ & $2.7 \pm 1.3$ & $2.8(1.7)$ & \\
\hline \multicolumn{5}{|c|}{ Stomach feeling heavy } \\
\hline 7 & $1(1.0)$ & $0(0.0)$ & $1(0.5)$ & 0.399 \\
\hline 6 & $5(5.0)$ & $4(4.1)$ & $9(4.5)$ & \\
\hline 5 & $13(12.9)$ & $16(16.3)$ & $29(14.6)$ & \\
\hline 4 & $16(15.8)$ & $23(23.5)$ & $39(19.6)$ & \\
\hline 3 & $25(24.8)$ & $20(20.4)$ & 45 (22.6) & \\
\hline 2 & $23(22.8)$ & $17(17.3)$ & $40(20.1)$ & \\
\hline 1 & $18(17.8)$ & $17(17.3)$ & 35 (17.6) & \\
\hline Mean $\pm \mathrm{SD}$ & $3.0 \pm 1.2$ & $3.2 \pm 1.2$ & $3.1(1.5)$ & \\
\hline \multicolumn{5}{|l|}{ Feeling queasy } \\
\hline 7 & $2(2.0)$ & $1(1.0)$ & $3(1.5)$ & 0.801 \\
\hline 6 & $4(4.0)$ & $4(4.1)$ & $8(4.0)$ & \\
\hline 5 & $6(6.0)$ & $5(5.1)$ & $11(5.5)$ & \\
\hline 4 & $11(10.9)$ & $9(9.2)$ & $20(10.1)$ & \\
\hline 3 & $9(8.9)$ & $11(11.2)$ & $20(10.1)$ & \\
\hline 2 & $19(18.8)$ & $18(18.4)$ & 37 (36.6) & \\
\hline 1 & $50(49.5)$ & $49(50.0)$ & 99 (98.0) & \\
\hline Mean \pm SD & $2.2 \pm 1.3$ & $2.2 \pm 1.2$ & $2.2(1.6)$ & \\
\hline
\end{tabular}

Table 2 continued

\begin{tabular}{|c|c|c|c|c|}
\hline $\begin{array}{l}\text { GOS scale } \\
\text { score }\end{array}$ & $\begin{array}{l}\text { Omeprazole } \\
(n=101)\end{array}$ & $\begin{array}{l}\text { Rabeprazole } \\
(n=98)\end{array}$ & $\begin{array}{l}\text { Total } \\
(N=199)\end{array}$ & $p$ value \\
\hline \multicolumn{5}{|l|}{ Burping } \\
\hline 7 & $1(1.0)$ & $1(1.0)$ & $2(1.0)$ & 0.387 \\
\hline 6 & $3(3.0)$ & $3(3.1)$ & $6(3.0)$ & \\
\hline 5 & $14(13.9)$ & $14(14.3)$ & $28(14.1)$ & \\
\hline 4 & $11(10.9)$ & $9(9.2)$ & $20(10.1)$ & \\
\hline 3 & $14(13.9)$ & $22(22.4)$ & $36(18.1)$ & \\
\hline 2 & $30(29.7)$ & $27(27.6)$ & $57(28.6)$ & \\
\hline 1 & $28(27.7)$ & $21(21.4)$ & $49(24.6)$ & \\
\hline Mean \pm SD & $2.7 \pm 1.3$ & $2.8 \pm 1.2$ & $2.7(1.5)$ & \\
\hline \multicolumn{5}{|l|}{ Early satiety } \\
\hline 7 & $3(3.0)$ & $0(0.0)$ & $3(1.5)$ & 0.092 \\
\hline 6 & $1(1.0)$ & $1(1.0)$ & $2(1.0)$ & \\
\hline 5 & $8(7.9)$ & $4(4.1)$ & $12(6.0)$ & \\
\hline 4 & $16(15.8)$ & $18(18.4)$ & $34(17.1)$ & \\
\hline 3 & $21(20.8)$ & $14(14.3)$ & 35 (17.6) & \\
\hline 2 & $24(23.8)$ & $23(23.5)$ & $47(23.6)$ & \\
\hline 1 & $28(27.7)$ & $37(37.8)$ & $65(32.7)$ & \\
\hline Mean \pm SD & $2.7 \pm 1.2$ & $2.3 \pm 1.1$ & $2.5(1.4)$ & \\
\hline \multicolumn{5}{|c|}{ Feeling of fullness } \\
\hline 7 & $3(3.0)$ & $0(0.0)$ & $3(1.5)$ & 0.565 \\
\hline 6 & $4(4.0)$ & $3(3.1)$ & $7(3.5)$ & \\
\hline 5 & $6(6.0)$ & $9(9.2)$ & $15(7.5)$ & \\
\hline 4 & $18(17.8)$ & $16(16.3)$ & $34(17.1)$ & \\
\hline 3 & $18(17.8)$ & $14(14.3)$ & $32(16.1)$ & \\
\hline 2 & $23(22.8)$ & $25(25.5)$ & $48(24.1)$ & \\
\hline 1 & $29(28.7)$ & $30(30.6)$ & 59 (29.6) & \\
\hline Mean \pm SD & $2.7 \pm 1.3$ & $2.6 \pm 1.3$ & $2.7(1.5)$ & \\
\hline
\end{tabular}

Data presented as $n(\%)$ unless otherwise indicated

Symptoms are evaluated on the 7-point GOS scale, from 1 ['no problem' (no symptoms)] to 7 ['very severe problem' (cannot be ignored and markedly limits my daily activities and often requires rest)]

GOS Global Overall Symptom, SD standard deviation

For upper gastrointestinal symptoms, sufficient relief after 2 and 4 weeks of PPI therapy was similar in the omeprazole $20 \mathrm{mg}$ and rabeprazole $10 \mathrm{mg}$ groups overall, and in patients with the homoEM and the heteroEM phenotypes (Fig. 6). In patients with the PM phenotype, a significantly greater proportion in the omeprazole $20 \mathrm{mg}$ group than in the rabeprazole $10 \mathrm{mg}$ group achieved sufficient relief of their upper gastrointestinal symptoms after 2 weeks ( 73.9 vs $26.3 \% ; p=0.002$ ), but differences were not statistically significant after 4 weeks (Fig. 6b).

Complete resolution of upper gastrointestinal symptoms was achieved in a significantly greater proportion of 


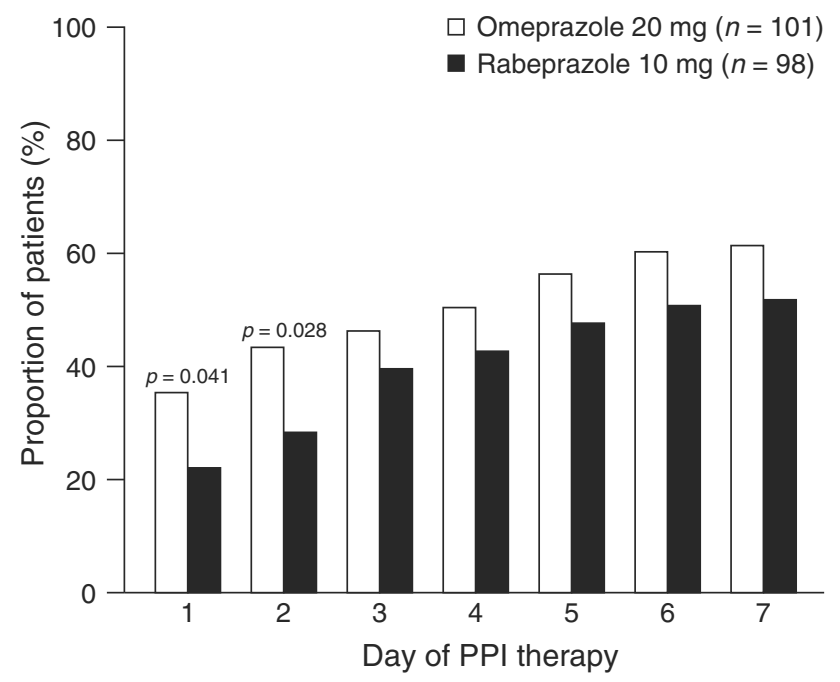

Fig. 2 Proportion of patients achieving sustained and sufficient relief of their reflux symptoms on days 1-7 of therapy with omeprazole $20 \mathrm{mg}$ or rabeprazole $10 \mathrm{mg}$. Sustained and sufficient symptom relief was defined as a GOS scale score of 1 or 2 that was maintained for at least 7 consecutive days. GOS Global Overall Symptom

patients in the omeprazole $20 \mathrm{mg}$ group than in the rabeprazole $10 \mathrm{mg}$ group after 4 weeks of PPI therapy (37.0 vs $20.8 \% ; p=0.013$ ), but differences were not statistically significant at the earlier, 2-week time point (Fig. 7a). When assessed according to CYP2C19 polymorphism, a significantly greater proportion of patients with the PM phenotype in the omeprazole $20 \mathrm{mg}$ group than in the rabeprazole $10 \mathrm{mg}$ group achieved complete resolution of their upper gastrointestinal symptoms after 4 weeks of PPI therapy (34.8 vs $21.1 \% ; p=0.033$ ), but no significant difference was observed at the earlier, 2-week time point (Fig. 7b).

\section{Safety}

Four adverse events (one each of itching, abdominal fullness, thirst and rash/exanthema) were reported in four patients treated with omeprazole $20 \mathrm{mg}$, of whom three had the homoEM and one the PM phenotype. All adverse events were considered by the investigator to be related to the study drug but none were graded as being serious. No adverse events were recorded for patients treated with rabeprazole $10 \mathrm{mg}$.

\section{Discussion}

In this 4-week, randomised study in Japanese patients with endoscopically confirmed reflux esophagitis, omeprazole $20 \mathrm{mg}$ once daily was significantly more effective than rabeprazole $10 \mathrm{mg}$ once daily at achieving early, sufficient, and sustained reflux symptom relief. On both day 1 and day 2 of PPI therapy, the proportion of patients achieving sufficient and sustained reflux symptom relief was at least $50 \%$ higher in the omeprazole $20 \mathrm{mg}$ group than in the rabeprazole $10 \mathrm{mg}$ group. The study thus met its primary efficacy endpoint of early onset of sufficient and sustained relief of reflux symptoms, defined as the first day of sufficient reflux symptom relief that was maintained for at least 7 consecutive days. Furthermore, a significantly greater proportion of patients achieved complete resolution of their reflux symptoms with omeprazole $20 \mathrm{mg}$ than with rabeprazole $10 \mathrm{mg}$ at both 2 and 4 weeks of PPI therapy, and a significantly greater proportion of patients treated with omeprazole $20 \mathrm{mg}$ also experienced complete resolution of upper gastrointestinal symptoms after 4 weeks of PPI therapy.

The pharmacodynamics and pharmacokinetics of PPIs are affected by genetic polymorphisms of CYP2C19, such that intragastric $\mathrm{pH}$ and plasma PPI levels are highest in patients with the PM phenotype, lowest in those with the homoEM phenotype and intermediate in those with the heteroEM phenotype [13]. Given that the PM phenotype is particularly prevalent in the Japanese population [13], we also evaluated outcomes based on CYP2C19 phenotype. At baseline, $21 \%$ of patients in our study were shown to have the PM phenotype, which is consistent with the prevalence for this trait in the general Japanese population (18.0-22.5\%) [13]. Our study showed that omeprazole $20 \mathrm{mg}$ was also more effective than rabeprazole $10 \mathrm{mg}$ at relieving and resolving reflux symptoms in patients with the PM phenotype; indeed, the proportion of patients achieving sufficient and sustained reflux symptom relief was at least $65 \%$ higher in the omeprazole $20 \mathrm{mg}$ group than in the rabeprazole $10 \mathrm{mg}$ group in patients with the PM phenotype on days 1-7 of PPI therapy, and this difference reached statistical significance on days 4-7. Reflux symptom relief remained significantly more effective with omeprazole $20 \mathrm{mg}$ than with rabeprazole $10 \mathrm{mg}$ at 2 and 4 weeks of PPI therapy in patients with the PM phenotype.

These results are consistent with the finding that CYP2C19 phenotype status has a greater effect on the pharmacokinetics of omeprazole than on the pharmacokinetics of rabeprazole, with a greater difference in systemic exposure to omeprazole than to rabeprazole seen across CYP2C19 phenotype categories [14, 23]. Although, by inference, it might be expected that systemic exposure to omeprazole is reduced in heteroEM and particularly homoEM phenotype patients, and this might translate into a decline in efficacy, our study did not show any significant differences in reflux-related outcomes favouring rabeprazole in these subgroups.

In line with these findings, results from a study conducted in healthy Japanese volunteers showed that early 

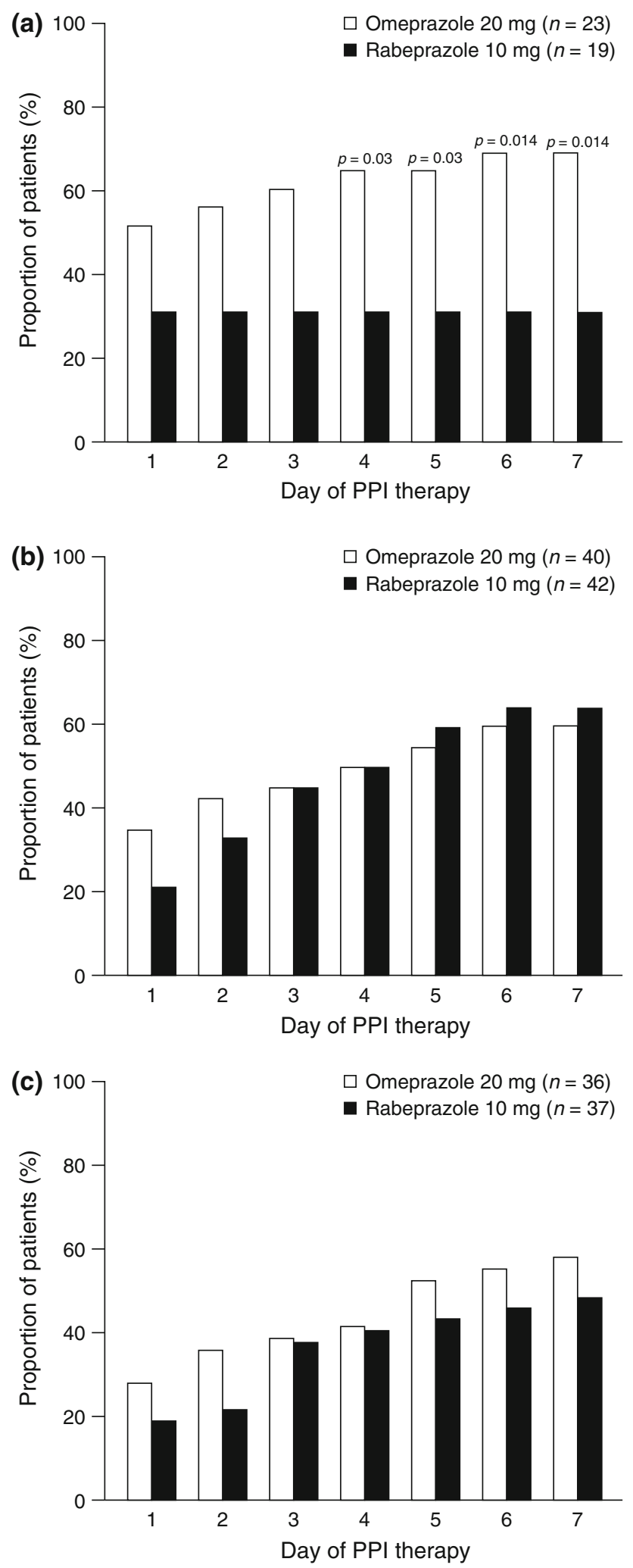

acid inhibition (measured by gastric $\mathrm{pH}$ ) was greater after omeprazole $20 \mathrm{mg}$ than after rabeprazole $10 \mathrm{mg}$ in a combined group of heteroEM and PM phenotypes, but not
Fig. 3 Proportion of patients achieving sustained and sufficient relief of their reflux symptoms on days 1-7 of therapy with omeprazole $20 \mathrm{mg}$ or rabeprazole $10 \mathrm{mg}$ in those with the CYP2C19 phenotype a PM, b heteroEM and $\mathbf{c}$ homoEM. Sustained and sufficient symptom relief was defined as a GOS scale score of 1 or 2 that was maintained for at least 7 consecutive days. CYP2C19 phenotype was unknown for two patients in the omeprazole group. CYP2C19 cytochrome P450 2C19, GOS Global Overall Symptom, heteroEM hetero extensive metaboliser, homoEM homo extensive metaboliser, $P M$ poor metaboliser

in homoEM phenotypes, although this significant difference did not persistent after 1 week of PPI therapy [17]. The results from our study suggest that these early differences in acid inhibition translate into improved, early reflux symptom relief with omeprazole $20 \mathrm{mg}$, compared with rabeprazole $10 \mathrm{mg}$, in patients with reflux esophagitis and the PM phenotype. However, early differences in acid inhibition seen with omeprazole $20 \mathrm{mg}$ versus rabeprazole $10 \mathrm{mg}$ in healthy volunteers with the homoEM or heteroEM phenotypes [15] may not translate into differences in early reflux symptom relief with the two PPIs. In our study, omeprazole $20 \mathrm{mg}$ and rabeprazole $10 \mathrm{mg}$ were broadly similarly effective at relieving and resolving reflux symptoms in patients with the homoEM and heteroEM phenotypes, with no significant differences observed at any of the time points analysed. Results from a multinational study conducted in Europe (where the prevalence of the PM phenotype is $\leq 3.7 \%$ [13]) showed a median time to heartburn relief of 1.5 days for both omeprazole $20 \mathrm{mg}$ and rabeprazole $20 \mathrm{mg}$ in patients with reflux esophagitis, suggesting that the two PPIs are similarly effective at early symptom relief in a population with predominantly homoEM and heteroEM phenotypes, even when the higher dose of $20 \mathrm{mg}$ rabeprazole is used [22]. Correspondingly, in another study conducted in Europe, decreases in 24-h esophageal acid exposure in response to PPI therapy in patients with GERD were similar with omeprazole $20 \mathrm{mg}$ and rabeprazole $20 \mathrm{mg}$ [24]. Taking into account the distribution of the different CYP2C19 phenotypes and the treatment doses, we consider it reasonable to expect that omeprazole $20 \mathrm{mg}$ might be more effective than rabeprazole $10 \mathrm{mg}$ at treating symptoms in Japanese patients with reflux esophagitis.

In addition to the typical symptoms of GERD (heartburn and acid regurgitation), patients with GERD commonly experience other upper gastrointestinal symptoms, including bloating, belching and abdominal pain [25]. Typical symptoms of GERD and dyspepsia-related symptoms often occur together, even in uninvestigated patients. Upper gastrointestinal symptoms have been shown to improve with PPI therapy [19]. The objective of our study was to evaluate improvement of symptoms in patients with reflux esophagitis, and the GOS scale, which measures upper 
(a) $\quad \square$ Omeprazole $20 \mathrm{mg}(n=101)$

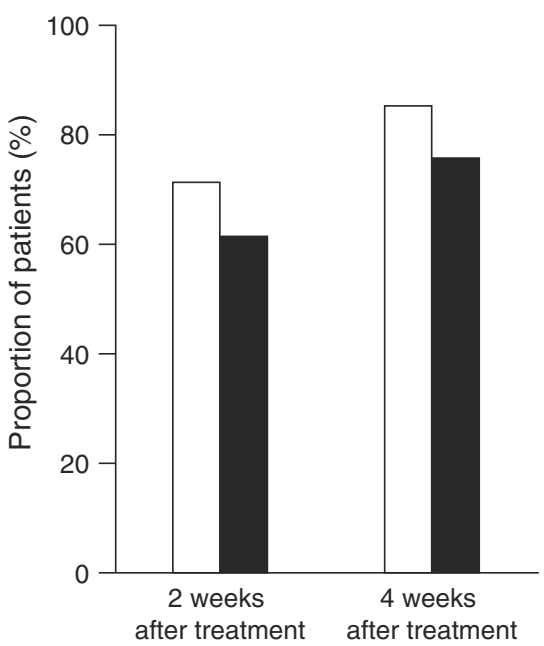

(b)

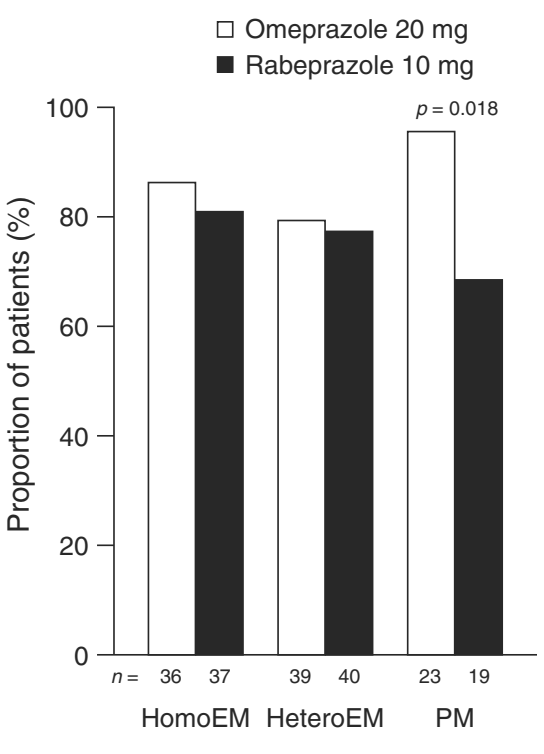

4 weeks after treatment
Fig. 4 Proportion of patients achieving sufficient relief of their reflux symptoms (GOS scale score of 1 or 2) after 2 and 4 weeks of therapy with omeprazole $20 \mathrm{mg}$ or rabeprazole $10 \mathrm{mg}$ a overall and b according to CYP2C19 phenotype. CYP2C19 phenotype was unknown for two patients in the omeprazole group. CYP2C19 cytochrome P450 2C19, GOS Global Overall Symptom, heteroEM hetero extensive metaboliser, homoEM homo extensive metaboliser, $P M$ poor metaboliser

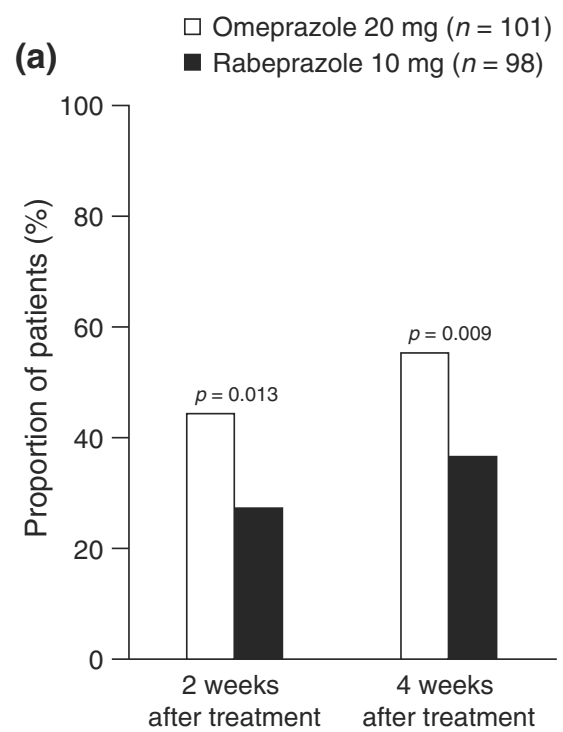

Fig. 5 Proportion of patients achieving complete resolution (GOS scale score of 1) of their reflux symptoms after 2 and 4 weeks of therapy with omeprazole $20 \mathrm{mg}$ or rabeprazole $10 \mathrm{mg}$ a overall and b according to $\mathrm{CYP} 2 \mathrm{C} 19$ phenotype. CYP2C19 phenotype was

gastrointestinal symptoms, was considered to be the appropriate questionnaire for this aim. When assessing a combination of eight upper gastrointestinal symptoms in this study, a significantly greater proportion of patients in the omeprazole $20 \mathrm{mg}$ group than in the rabeprazole $10 \mathrm{mg}$ (b)

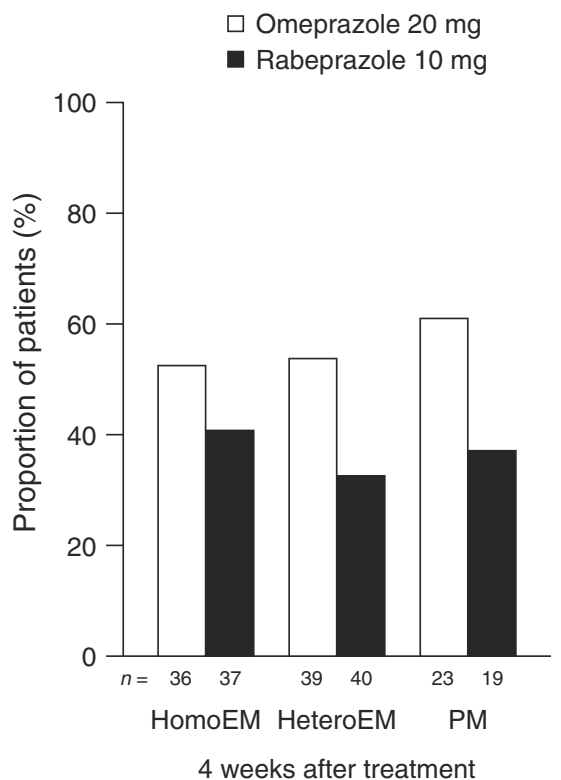

unknown for two patients in the omeprazole group. CYP2C19 cytochrome P450 2C19, GOS Global Overall Symptom, heteroEM hetero extensive metaboliser, homoEM homo extensive metaboliser, $P M$ poor metaboliser

group experienced complete symptom resolution at 4 weeks of PPI therapy. In patients with the PM phenotype, omeprazole $20 \mathrm{mg}$ showed a significantly higher efficacy than rabeprazole $10 \mathrm{mg}$ at relieving (at 2 weeks) and resolving (at 4 weeks) upper gastrointestinal symptoms. 


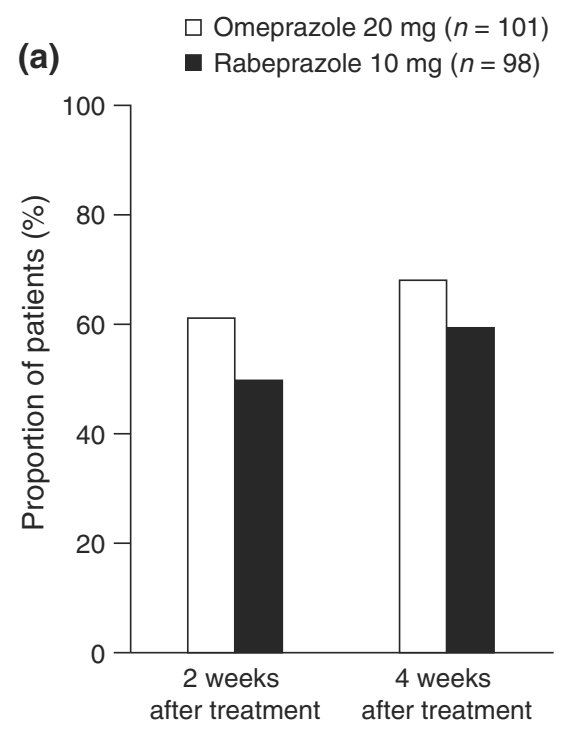

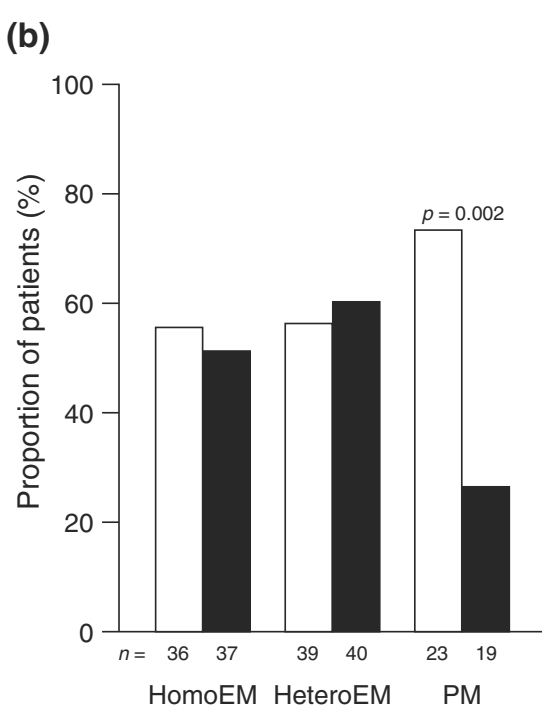

2 weeks after treatment

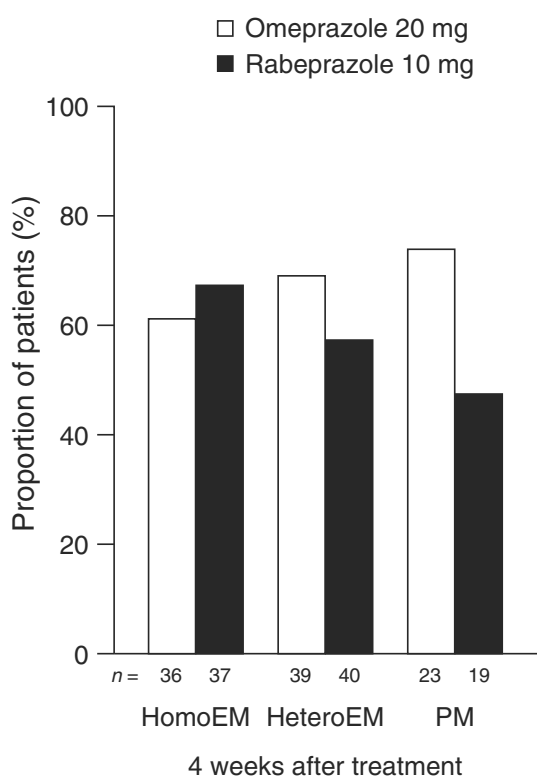

Fig. 6 Proportion of patients achieving sufficient relief (GOS scale score of 1 or 2) of their upper gastrointestinal symptoms after 2 and 4 weeks of therapy with omeprazole $20 \mathrm{mg}$ or rabeprazole $10 \mathrm{mg}$ a overall and b according to CYP2C19 phenotype. CYP2C19 phenotype was unknown for two patients in the omeprazole group. CYP2C19 cytochrome P450 2C19, GOS Global Overall Symptom, heteroEM hetero extensive metaboliser, homoEM homo extensive metaboliser, $P M$ poor metaboliser

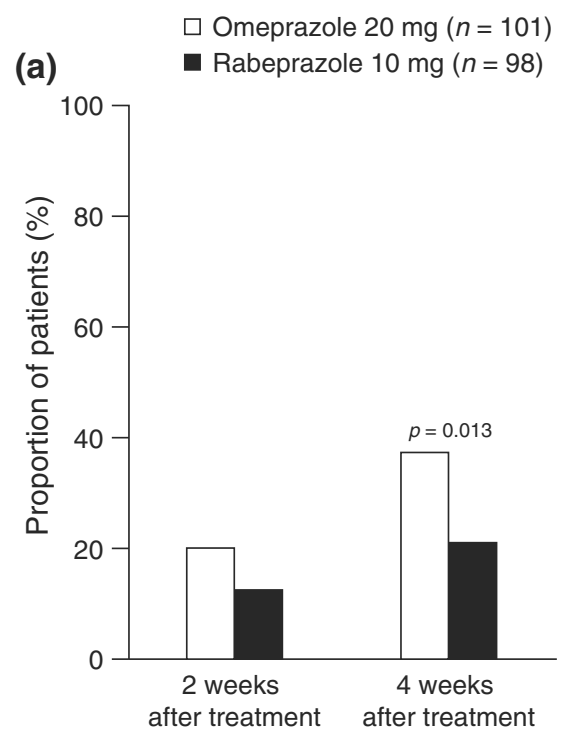

(a) (b)

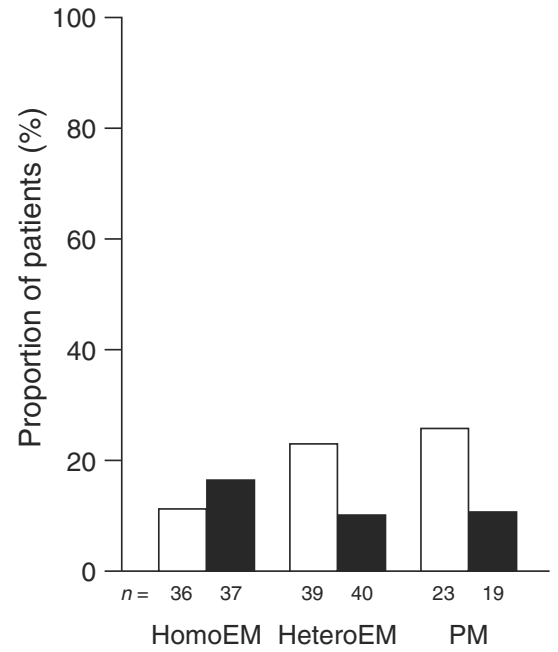

2 weeks after treatment

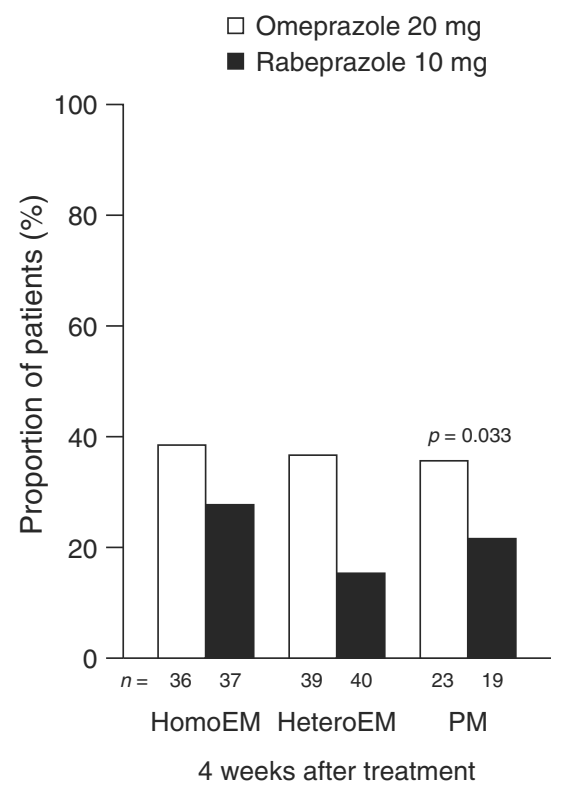

Fig. 7 Proportion of patients achieving complete resolution (GOS scale score of 1) of their upper gastrointestinal symptoms after 2 and 4 weeks of therapy with omeprazole $20 \mathrm{mg}$ or rabeprazole $10 \mathrm{mg}$ a overall and b according to CYP2C19 phenotype was unknown for

Furthermore, omeprazole $20 \mathrm{mg}$ and rabeprazole $10 \mathrm{mg}$ were broadly similarly effective at relieving and resolving these symptoms in patients with the homoEM and heteroEM phenotypes. Our results therefore suggest that improved early symptom relief with omeprazole $20 \mathrm{mg}$, compared with rabeprazole $10 \mathrm{mg}$, in patients with reflux two patients in the omeprazole group. CYP2C19 cytochrome P450 2C19, GOS Global Overall Symptom, heteroEM hetero extensive metaboliser, homoEM homo extensive metaboliser, $P M$ poor metaboliser

esophagitis and the PM phenotype applies not only to reflux symptoms, but also to other upper gastrointestinal symptoms that are common in GERD.

Both omeprazole $20 \mathrm{mg}$ and rabeprazole $10 \mathrm{mg}$ were generally well-tolerated in this 4-week study. Only four adverse events were recorded (itching, abdominal fullness, 
thirst, rash/exanthema), all in patients receiving omeprazole $20 \mathrm{mg}$. Although the incidence of adverse events was low, there was no evidence of a relationship with the CYP2C19 phenotype.

Key strengths of our study include the use of a validated patient-reported outcome instrument to record symptoms, the inclusion of patients with endoscopically confirmed reflux esophagitis, the separate analyses according to all three CYP2C19 phenotypes, and the use of study drug doses relevant to clinical practice in Japan. A limitation of our study is the open-label design, which means that confounding of outcomes by treatment expectation cannot be excluded. In addition, the symptomatic improvements seen with omeprazole $20 \mathrm{mg}$ over rabeprazole $10 \mathrm{mg}$ were not corroborated by endoscopic evaluation of reflux esophagitis. Since there has been little published data regarding symptom-based evaluations in early-phase response to treatment in GERD [26-29], the primary endpoint of this study was symptom relief in the early phase of treatment rather than mucosal healing, which is why endoscopy was not included in the study protocol. Regarding 4-8-week initial therapies for reflux esophagitis, symptom relief was relevant to mucosal healing [30]. Although endoscopy should be performed to confirm cure of reflux esophagitis, the fact that many patients achieved sufficient relief of reflux symptoms at 4 weeks in this study indicates that many of them might be cured. Furthermore, although doses of omeprazole $20 \mathrm{mg}$ and rabeprazole $10 \mathrm{mg}$ were consistent with those approved for current clinical practice in Japan, evidence suggests that rabeprazole has a more potent effect on acid suppression than omeprazole on a mg for $\mathrm{mg}$ basis [31]. It is open to question whether the advantage for omeprazole $20 \mathrm{mg}$ would have been sustained if rabeprazole had been administered at the same dose; however, doses were chosen based on current clinical practice in Japan.

In conclusion, the results of our study show that omeprazole $20 \mathrm{mg}$ once-daily is significantly more effective than rabeprazole $10 \mathrm{mg}$ once daily at achieving sufficient and sustained relief of reflux symptom in the first 2 days of PPI therapy in Japanese patients with reflux esophagitis. This significant advantage of omeprazole $20 \mathrm{mg}$ therapy also extends to resolution of reflux and upper gastrointestinal symptoms. Omeprazole $20 \mathrm{mg}$ is more effective than rabeprazole $10 \mathrm{mg}$ at early relief and resolution of reflux symptoms and other upper gastrointestinal symptoms in patients with the PM phenotype, and has similar effectiveness to rabeprazole $10 \mathrm{mg}$ in patients with the heteroEM and homoEM phenotypes. These findings can assist physicians with disease management in patients with reflux esophagitis in Japan, where the PM phenotype is much more common than in the USA or Europe [13].
Acknowledgments We would like to thank the following study investigators: Kiyoharu Ito (Ozaki Clinic), Tetsuya Murao (Ozaki Clinic), Satoshi Yamada (Yamada Clinic), Akihiko Kurosawa (Kurosawa Gastroenterological Clinic), Toshio Murai (Murai Clinic), Yukihiro Sakurai (Sakurai Digestive Disease Clinic), Shuhei Tazaki (Tazaki Clinic) and Ryuzo Murai (Onaka Clinic).

Conflict of interest The authors declare that they have no conflict of interest.

Open Access This article is distributed under the terms of the Creative Commons Attribution Noncommercial License which permits any noncommercial use, distribution, and reproduction in any medium, provided the original author(s) and the source are credited.

\section{References}

1. Donnellan C, Sharma N, Preston C, Moayyedi P. Medical treatments for the maintenance therapy of reflux oesophagitis and endoscopic negative reflux disease. Cochrane Database Syst Rev. 2005;2:CD003245.

2. Vakil N, Veldhuyzen van Zanten S, Kahrilas P, Dent J, Jones R. The Montreal definition and classification of gastro-esophageal reflux disease (GERD) — a global evidence-based consensus. Am J Gastroenterol. 2006;101:1900-20.

3. Kinoshita Y, Adachi K, Hongo M, Haruma K. Systematic review of the epidemiology of gastroesophageal reflux disease in Japan. J Gastroenterol. 2011;46:1092-103.

4. Matsuki N, Fujita T, Watanabe N, et al. Lifestyle factors associated with gastroesophageal reflux disease in the Japanese population. J Gastroenterol. 2013;48:340-9.

5. Ronkainen J, Aro P, Storskrubb T, et al. Gastro-oesophageal reflux symptoms and health-related quality of life in the adult general population-the Kalixanda study. Aliment Pharmacol Ther. 2006;23:1725-33.

6. Wahlqvist P, Reilly M, Barkun AN. Systematic review: the impact of gastro-oesophageal reflux disease on work productivity. Aliment Pharmacol Ther. 2006;24:259-72.

7. Wahlqvist $\mathrm{P}$, Karlsson M, Johnson D, Carlsson J, Bolge S, Wallander MA. Relationship between symptoms of gastroesophageal reflux disease and costs: a database study in a US cohort. Value Health. 2007;10:A149.

8. Wiklund I, Carlsson J, Vakil N. Gastroesophageal reflux symptoms and well-being in a random sample of the general population of a Swedish community. Am J Gastroenterol. 2006;101:18-28.

9. Ronkainen J, Talley NJ, Storskrubb T, et al. Erosive esophagitis is a risk factor for Barrett's esophagus: a community-based endoscopic follow-up study. Am J Gastroenterol. 2011;106:1946-52.

10. van Pinxteren B, Numans ME, Bonis PA, Lau J. Short-term treatment with proton pump inhibitors, $\mathrm{H}_{2}$-receptor antagonists and prokinetics for gastro-oesophageal reflux disease-like symptoms and endoscopy negative reflux disease. Cochrane Database Syst Rev. 2004;4:CD002095.

11. Kulig M, Leodolter A, Vieth M, et al. Quality of life in relation to symptoms in patients with gastro-oesophageal reflux disease-an analysis based on the ProGERD initiative. Aliment Pharmacol Ther. 2003;18:767-76.

12. Pace F, Negrini C, Wiklund I, Rossi C, Savarino V. Quality of life in acute and maintenance treatment of non-erosive and mild erosive gastro-oesophageal reflux disease. Aliment Pharmacol Ther. 2005;22:349-56.

13. Furuta T, Sugimoto M, Shirai N, Ishizaki T. CYP2C19 pharmacogenomics associated with therapy of Helicobacter pylori infection and gastro-esophageal reflux diseases with a proton pump inhibitor. Pharmacogenomics. 2007;8:1199-210. 
14. Shirai N, Furuta T, Moriyama Y, et al. Effects of CYP2C19 genotypic differences in the metabolism of omeprazole and rabeprazole on intragastric pH. Aliment Pharmacol Ther. 2001;15: 1929-37.

15. Saitoh T, Fukushima Y, Otsuka H, et al. Effects of rabeprazole, lansoprazole and omeprazole on intragastric $\mathrm{pH}$ in CYP2C19 extensive metabolizers. Aliment Pharmacol Ther. 2002;16: 1811-7.

16. Yamagishi H, Koike T, Ohara S, et al. Early effects of Lansoprazole orally disintegrating tablets on intragastric $\mathrm{pH}$ in CYP2C19 extensive metabolizers. World J Gastroenterol. 2008; 14:2049-54.

17. Furuta K, Adachi K, Ohara S, et al. Relationship between the acid-inhibitory effects of two proton pump inhibitors and CYP2C19 genotype in Japanese subjects: a randomized two-way crossover study. J Int Med Res. 2010;38:1473-83.

18. Veldhuyzen Van Zanten SJ, Chiba N, Armstrong D, et al. Validation of a 7-point global overall symptom scale to measure the severity of dyspepsia symptoms in clinical trials. Aliment Pharmacol Ther. 2006;23:521-9.

19. Sakurai K, Nagahara A, Inoue K, et al. Efficacy of omeprazole, famotidine, mosapride and teprenone in patients with upper gastrointestinal symptoms: an omeprazole-controlled randomized study (J-FOCUS). BMC Gastroenterol. 2012;12:42.

20. Veldhuyzen van Zanten S, Armstrong D, Chiba N, et al. Esomeprazole $40 \mathrm{mg}$ once a day in patients with functional dyspepsia: the randomized, placebo-controlled "ENTER" trial. Am J Gastroenterol. 2006;101:2096-106.

21. Dewan B, Philipose N. Lafutidine $10 \mathrm{mg}$ versus rabeprazole 20 $\mathrm{mg}$ in the treatment of patients with heartburn-dominant uninvestigated dyspepsia: a randomized, multicentric trial. Gastroenterol Res Pract. 2011;2011:640685.

22. Bytzer P, Morocutti A, Kennerly P, Ravic M, Miller N. Effect of rabeprazole and omeprazole on the onset of gastro-oesophageal reflux disease symptom relief during the first seven days of treatment. Scand J Gastroenterol. 2006;41:1132-40.

23. Furuta T, Ohashi K, Kosuge K, et al. CYP2C19 genotype status and effect of omeprazole on intragastric $\mathrm{pH}$ in humans. Clin Pharmacol Ther. 1999;65:552-61.

24. Galmiche JP, Zerbib F, Ducrottè P, et al. Decreasing oesophageal acid exposure in patients with GERD: a comparison of rabeprazole and omeprazole. Aliment Pharmacol Ther. 2001;15:1343-50.

25. Vakil N, Halling K, Wernersson B, Ohlsson L. Rome III functional dyspepsia criteria show substantial symptom overlap with gastroesophageal reflux disease. Gut. 2011;60(Suppl 3):A154.

26. Fock KM, Teo EK, Ang TL, Chua TS, Ng TM, Tan YL. Rabeprazole vs esomeprazole in non-erosive gastro-esophageal reflux disease: a randomized, double-blind study in urban Asia. World J Gastroenterol. 2005;11:3091-8.

27. Archimandritis AJ, Nikolopoulou V, Kouklakis G, Paraskevas E, Avgerinos A, Tsianos E, Triantafillidis JK, Hellenic Rabeprazole Study Group. Effects of rabeprazole on early symptom relief in gastro-oesophageal reflux disease: the Hellenic Rabeprazole Study Group surveillance study. Curr Med Res Opin. 2005;21: 603-10.

28. Mee AS, Rowley JL. Rapid symptom relief in reflux oesophagitis: a comparison of lansoprazole and omeprazole. Aliment Pharmacol Ther. 1996;10:757-63.

29. Tominaga K, Iwakiri R, Fujimoto K, GERD 4 Study Group, et al. Rikkunshito improves symptoms in PPI-refractory GERD patients: a prospective, randomized, multicenter trial in Japan. J Gastroenterol. 2012;47:284-92.

30. Khan M, Santana J, Donnellan C, Preston C, Moayyedi P. Medical treatments in the short term management of reflux oesophagitis. Cochrane Database Syst Rev. 2007;2:CD003244.

31. Stedman CA, Barclay ML. Review article: comparison of the pharmacokinetics, acid suppression and efficacy of proton pump inhibitors. Aliment Pharmacol Ther. 2000;14:963-78. 